\title{
Growth-Associated Protein 43 Is Located in Type I Corticothalamic Terminals in the Cat Visual Thalamus
}

\author{
Martha E. Bickford \\ Department of Anatomical Sciences and Neurobiology, University of Louisville, School of Medicine, Louisville, \\ Kentucky 40292
}

Growth-associated protein 43 (GAP 43) is a presynaptic protein that has been proposed to be involved in synaptic plasticity. To determine the location of GAP 43 within the synaptic circuitry of the thalamus, immunocytochemical staining for GAP 43 was examined in a relay nucleus, the dorsal lateral geniculate nucleus (dLGN), and two association nuclei, the pulvinar nucleus and the lateral subdivision of the lateral posterior (LP) nucleus. In the dLGN, moderate neuropil staining was seen in the $A$ laminae, and denser staining was found in the interlaminar zones and the $\mathrm{C}$ laminae. Uniform dense staining of the neuropil was found in both the pulvinar and LP nuclei. At the ultrastructural level, the GAP 43 staining was restricted to small-diameter myelinated axons, thin unmyelinated fibers, and small terminals that contained densely packed round vesicles (RS profiles) and made asymmetric synaptic contacts with

Growth-associated protein 43 (GAP 43) is expressed widely in the developing brain and is involved in the establishment of synaptic connections (Benowitz and Perrone-Bizzozero, 1991; Benowitz and Routtenberg, 1997). GAP 43 expression decreases during development, but significant levels continue to be present in the adult brain, where it has been proposed to be involved in synaptic plasticity (Benowitz et al., 1988; Neve et al., 1988; Benowitz and Perrone-Bizzozero, 1991; Benowitz and Routtenberg, 1997). For example, GAP 43 may mediate an activity-dependent enhancement of neurotransmitter release. It has been shown that GAP 43 is dissociated from calmodulin and phosphorylated in an activity-dependent manner. Phosphorylated GAP 43 can then enhance the release of neurotransmitters from synaptic vesicles (De Graan et al., 1990; Benowitz and Routtenberg, 1997). It has previously been noted that immunocytochemical staining for GAP 43 in the thalamus and cortex is denser in association areas than in primary sensory areas (Benowitz et al., 1988; Benowitz and Perrone-Bizzozero, 1991; Neve et al., 1988; Benowitz and Routtenberg, 1997). This differential staining pattern is thought to reflect a greater potential for synaptic plasticity within association areas.

The key distinction between relay and association nuclei of the thalamus appears to lie in their connections with the cortex. Although all dorsal thalamic nuclei are innervated by small type

Received March 26, 1999; revised June 25, 1999; accepted June 30, 1999.

This work was supported by National Institute of Neurological Diseases and Stroke Grant R29NS35377 and National Science Foundation Grant 9728089. I thank Martin Boyce, Michael Eisenback, and Cathie Caple for expert technical assistance.

Correspondence should be addressed to Martha E. Bickford, Department of Anatomical Sciences and Neurobiology, University of Louisville, School of Medicine, 500 South Preston Street, Louisville, KY 40292.

Copyright (C) 1999 Society for Neuroscience $\quad 0270-6474 / 99 / 190001-\bullet \$ 05.00 / 0$ small-caliber dendrites in the extraglomerular neuropil. The distribution of immunocytochemical label within the visual thalamus suggests that GAP 43 is confined to type I corticothalamic terminals and axons that originate from extrastriate cortical areas. These results also suggest that in both relay and association nuclei GAP 43 may be used to augment the cortical control of thalamic activity. In addition, these results underscore the distinction between the small type I corticothalamic terminals, which appear to contain GAP 43 throughout the visual thalamus, and the large type II corticothalamic terminals that, like the type II retinal terminals in the dLGN, do not contain GAP 43.

Key words: lateral geniculate nucleus; pulvinar nucleus; lateral posterior nucleus; relay; association; ultrastructure; synaptic plasticity

I corticothalamic terminals, only the association nuclei receive additional large type II corticothalamic terminals (Jones and Powell, 1969; Mathers, 1972; Robson and Hall, 1977; Ogren and Hendrickson, 1979; Vidnyánszky and Hámori, 1994; Ojima et al., 1996; Rockland, 1996; Vidnyánszky et al., 1996; Erişir et al., 1997; Bickford et al., 1998; Feig and Harting, 1998). Based on comparisons of the circuitry of thalamic relay and association nuclei, it has been proposed that type II corticothalamic terminals are functionally equivalent to the type II primary sensory terminals that innervate the relay nuclei. This has led to the idea that although relay nuclei modulate the flow of sensory information from the periphery to the cortex, association nuclei gate the passage of information from one cortical area to another (Guillery, 1995; Sherman and Guillery, 1996; Patel and Bickford, 1997; Bickford et al., 1998; Feig and Harting, 1998; Patel et al., 1999).

To determine where GAP 43 is located within the synaptic circuitry of relay and association nuclei of the thalamus, the distribution of immunocytochemical labeling for GAP 43 was

This article is published in The Journal of Neuroscience, Rapid Communications Section, which publishes brief, peerreviewed papers online, not in print. Rapid Communications are posted online approximately one month earlier than they would appear if printed. They are listed in the Table of Contents of the next open issue of JNeurosci. Cite this article as: JNeurosci, 1999, 19:RC22 (1-5). The publication date is the date of posting online at www.jneurosci.org.

http://www.jneurosci.org/cgi/content/full/3407 
Figure 1. GAP 43 staining is denser in thalamic association nuclei than in thalamic relay nuclei. At low magnification $(A)$ it can be seen that GAP 43 staining in the pulvinar nucleus (PUL) and the medial $(L P m)$ and lateral $(L P l)$ divisions of the lateral posterior nucleus is denser than in the A lamina $(A, A 1)$ of the dLGN. All GAP 43 staining in the visual thalamus is confined to the neuropil. Somata show no GAP 43 staining but are prominently outlined by dense GAP 43 -stained profiles. In the dLGN, moderate staining is seen in the A laminae, and denser staining is found in the interlaminar zones $(B, C)$. The arrow points to an area shown at higher magnification in $C$. GAP 43 staining is dense in the dLGN C laminae $(D)$ and pulvinar $(E)$ and $\mathrm{LPl}(F)$ nuclei. Scale bars: $A, 1 \mathrm{~mm} ; B, 100 \mu \mathrm{m} ; C, 50 \mu \mathrm{m}$ (also applies to $D-F)$. OT, Optic tract.
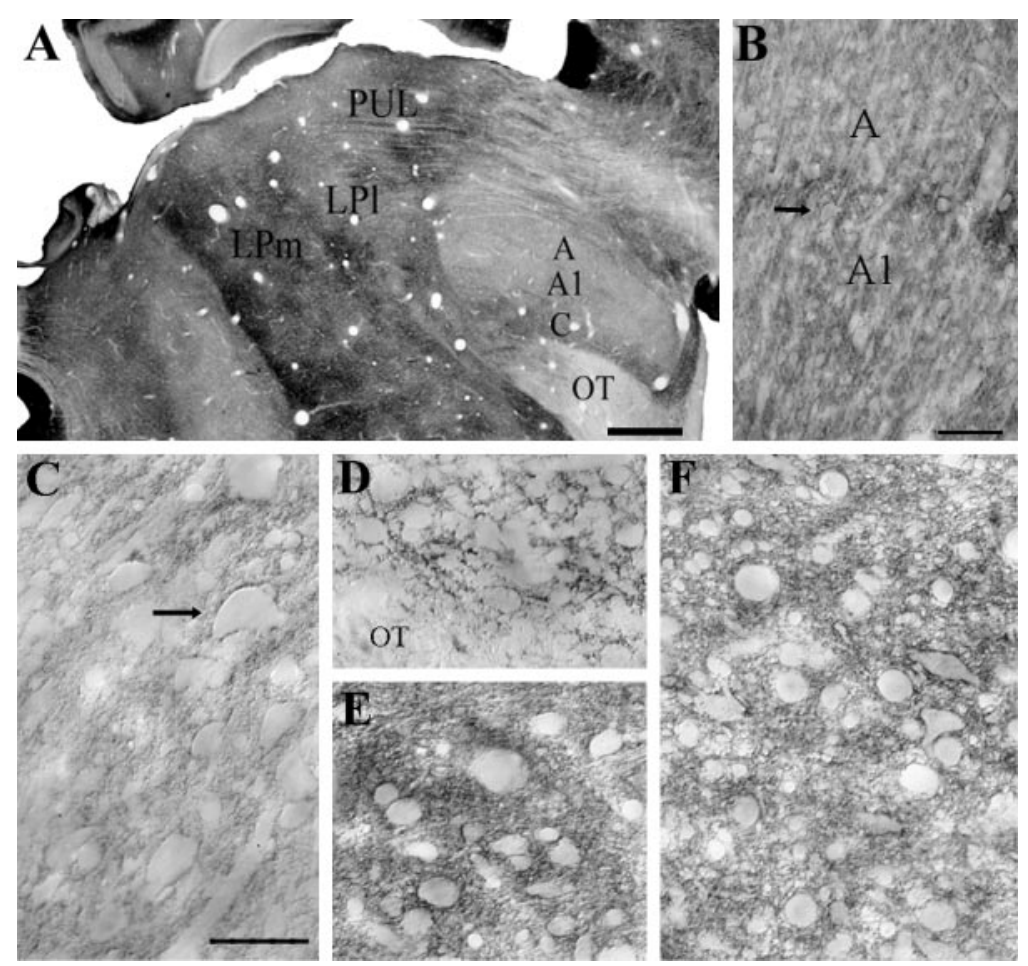

examined in the visual thalamus of the cat. GAP 43 staining was examined in a well characterized relay nucleus, the dorsal lateral geniculate nucleus (dLGN), and two association nuclei, the pulvinar nucleus and the lateral subdivision of the lateral posterior (LP) nucleus.

\section{MATERIALS AND METHODS}

Three adult cats were used for this study. The cats were deeply anesthetized and perfused with $4 \%$ paraformaldehyde, $4 \%$ paraformaldehyde, and $0.5 \%$ glutaraldehyde or $2 \%$ paraformaldehyde and $0.1 \%$ glutaraldehyde in $0.1 \mathrm{~m}$ sodium phosphate buffer, $\mathrm{pH}$ 7.4. The brains were removed, and blocks of the thalamus were sectioned in the sagittal or coronal plane with a vibratome to a thickness of $50 \mu \mathrm{m}$. Sections were stained with the GAP 43 antibody (mouse monoclonal; Boehringer Mannheim, Indianapolis, IN) diluted 1:1000-1:2000. Using previous immunocytochemical techniques (Patel and Bickford, 1997; Patel et al., 1999), the antibody was tagged with a biotinylated goat-anti-mouse antibody, a complex of avidin and biotinylated horseradish peroxidase, and revealed with a diaminobenzidine reaction. Sections were then mounted on slides for light microscopy or processed for electron microscopy as previously described (Patel and Bickford, 1997; Patel et al., 1999).

\section{RESULTS}

As shown in Figure 1A, GAP 43 staining is denser in the pulvinar and LP nuclei than in the dLGN. At higher magnification (Fig. $1 B-F)$, it can be seen that in all three nuclei, GAP 43 staining is confined to the neuropil. As previously noted (Baekelandt et al., 1994), in the dLGN moderate staining is seen in the A laminae, and denser staining is found in the interlaminar zones (Fig. $1 B, C$ ), the C laminae (Fig. 1D), and the medial interlaminar nucleus. Uniform dense staining of the neuropil is found in both the pulvinar (Fig. $1 E$ ) and LP (Fig. $1 F$ ) nuclei. In all three nuclei, somata show no GAP 43 staining but are prominently outlined by dense GAP 43-stained profiles.

GAP 43 staining was examined at the ultrastructural level in blocks of tissue from the dLGN (A lamina), pulvinar nucleus, and lateral LP nucleus. In each nucleus, the morphology of profiles stained for GAP 43 was consistent with the morphology of type I corticothalamic terminals and axons. As illustrated in Figure 2, in all three nuclei, GAP 43 staining was located in small terminals that contained densely packed round vesicles (RS profiles) and contacted dendrites with thick postsynaptic densities. Other GAP 43-stained profiles included small-diameter myelinated axons and thin unmyelinated fibers that formed bundles, which were particularly abundant surrounding somata (Fig. 3). Minimal GAP 43 staining was observed in dendrites, somata, or terminals other than those exhibiting RS morphology. The majority of GAP 43 staining was excluded from glomerular arrangements, where type II cortical or retinal terminals are abundant (Fig. 4).

In each nucleus, photographs were taken of synaptic contacts made by GAP 43-stained terminals (dLGN, $n=104$; pulvinar nucleus, $n=89$; lateral LP nucleus, $n=54$ ). Of the sampled contacts, the vast majority of GAP 43 -stained terminals contacted small-caliber dendrites in the extraglomerular neuropil (235 of 247 or $95 \%$ ). These presumably distal dendrites were often contacted by other labeled or unlabeled RS profiles (Fig. 2). These synaptic arrangements are characteristic of type I corticothalamic terminals in the cat visual thalamus (Jones and Powell, 1969; Vidnyánszky and Hámori, 1994; Erişir et al., 1997; Bickford et al., 1998).

\section{DISCUSSION}

In the visual thalamus, the morphology and synaptic arrangements of GAP 43-stained profiles are identical to the morphology and synaptic arrangements of type I corticothalamic terminals. That is, GAP 43 staining was confined to RS profiles that contacted small-caliber dendrites in the extraglomerular neuropil. The other main source of RS profiles in the visual thalamus is the cholinergic parabrachial region (PBR) of the brainstem. However, in the dLGN and pulvinar nucleus, PBR terminals primarily innervate glomeruli (Cucchiaro et al., 1988; Erişir et al., 1997; Patel and Bickford, 1997). Because GAP 43 staining was for the most part excluded from glomeruli, it is unlikely that GAP 43 is 

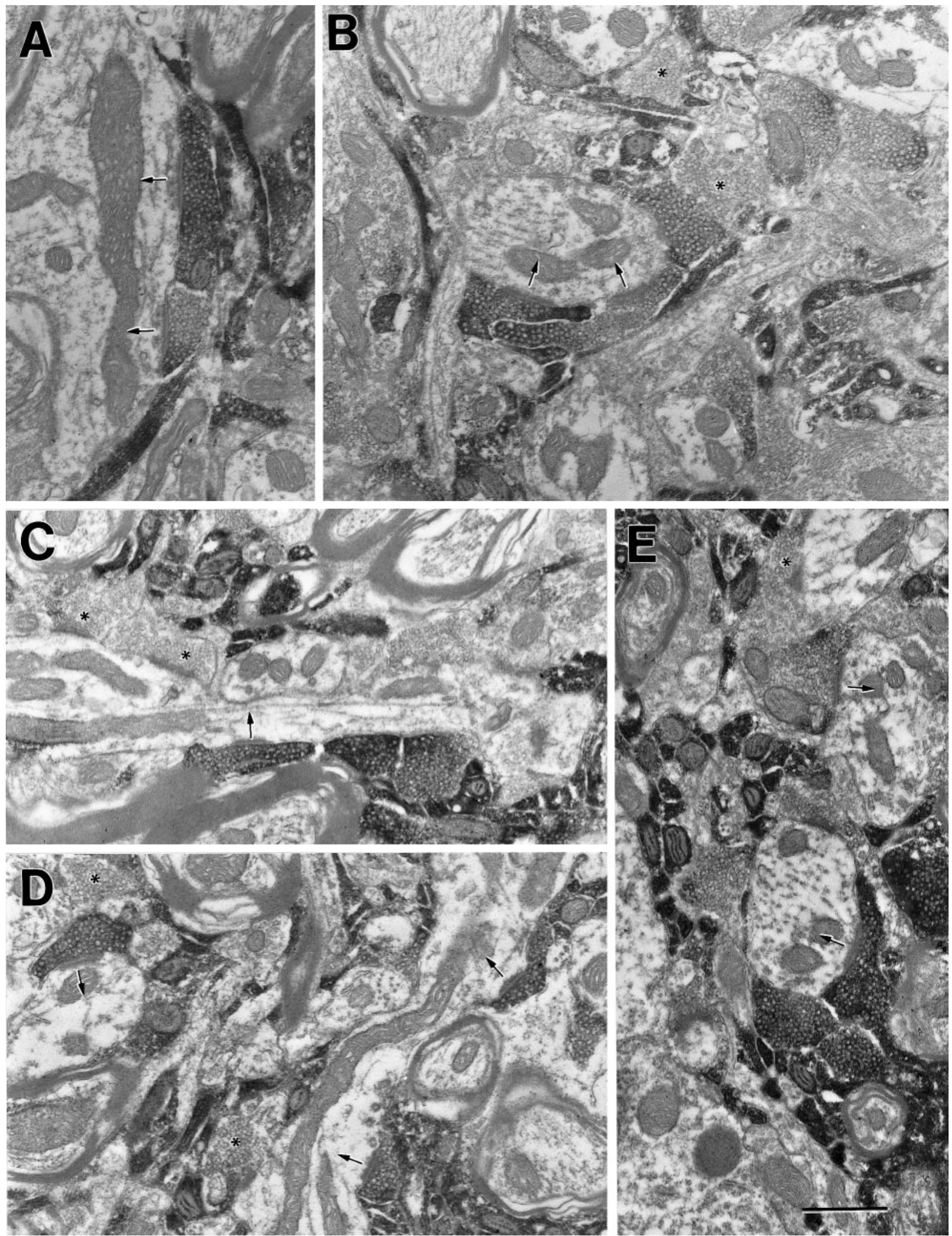

Figure 2. In the visual thalamus, GAP 43 staining is located in small terminals that contain densely packed round vesicles (RS profiles) and contact (arrows) dendrites with thick postsynaptic densities. Some RS profiles are not labeled by the GAP 43 antibody (asterisks). A, B, GAP 43 staining in the lateral geniculate nucleus. $C, E$, GAP 43 staining in the lateral posterior nucleus. $D$, GAP 43 staining in the pulvinar nucleus. Scale bar, $0.5 \mu$ m. 


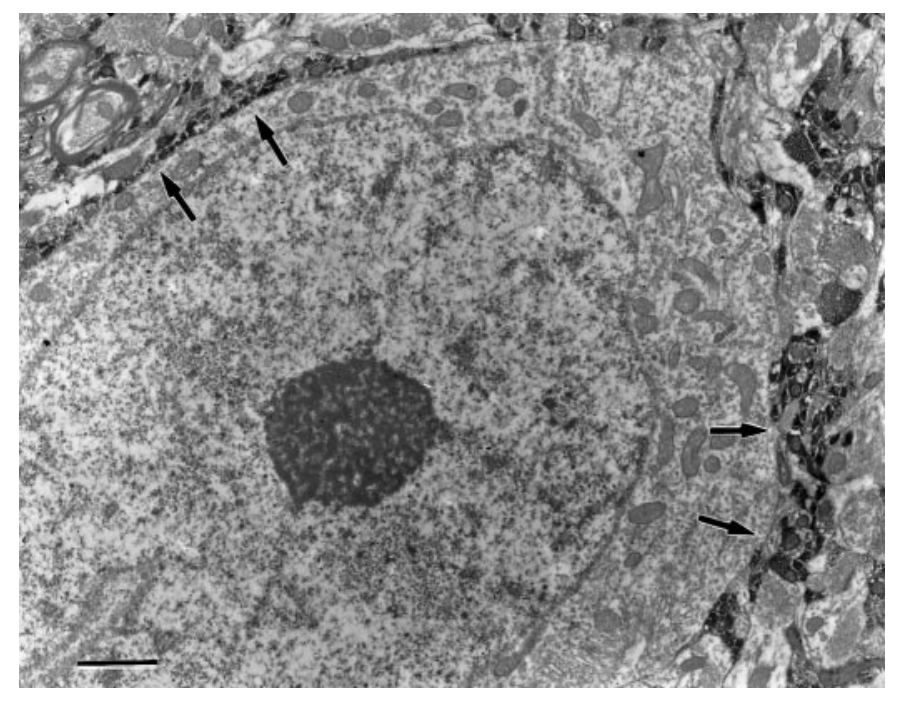

Figure 3. GAP 43 staining is abundant in small unmyelinated fibers that surround somata (arrows). Scale bar, $1 \mu \mathrm{m}$.

contained in PBR terminals. In addition, although PBR terminals in the lateral LP nucleus are located outside of glomeruli, they make up a very small proportion of the RS terminals in this region of the thalamus (Patel et al., 1999). Because GAP 43-stained terminals are quite dense in the lateral LP nucleus, it is likely that most, if not all, GAP 43 is contained within corticothalamic axons and terminals.

The distribution of GAP 43 staining in the visual thalamus correlates well with the distribution of certain groups of corticothalamic terminals. In the dLGN, GAP 43 staining is densest in the interlaminar zones, where it has been noted that corticothalamic terminals from area 18 are most dense (Updyke, 1975; Vidnyánszky and Hámori, 1994). GAP 43 staining is also quite dense in the $\mathrm{C}$ laminae, which receive the majority of their cortical input from areas 18 and 19 (Updyke, 1975). Because not all RS profiles were labeled by the GAP 43 antibody, perhaps the reason that thalamic association nuclei are more densely stained for GAP 43 is that this protein is preferentially distributed within type I terminals from extrastriate cortical areas. In support of this idea, in the human striate cortex, GAP 43 mRNA is found only in superficial layer cells, but in the inferotemporal cortex, GAP 43 mRNA is additionally found in deeper cortical layers (Neve et al., 1988) where corticothalamic neurons are located (Gilbert and Kelly, 1975; Abramson and Chalupa, 1985).

Regardless of the precise cortical area of origin, the present results suggest that in the visual thalamus, GAP 43 is located exclusively in type I corticothalamic terminals. This distribution may account for the synaptic potentiation observed after highfrequency stimulation of the optic radiations in dLGN slices (Scharfman et al., 1990; McCormick and von Krosigk, 1992). High-frequency stimulation of corticothalamic fibers could result in the phosphorylation of the GAP 43 contained within these terminals, which might lead to the release of a larger volume of glutamate from their synaptic vesicles after subsequent stimulation. It is also interesting to note that a calcium-calmodulindependent protein kinase (CaM kinase II) is located postsynaptic to many, but not all, RS profiles (Liu and Jones, 1996). It has been proposed that $\mathrm{CaM}$ kinase II plays an important role in the process of long-term potentiation (Malenka et al., 1989). In addition, certain types of metabotropic glutamate receptors appear
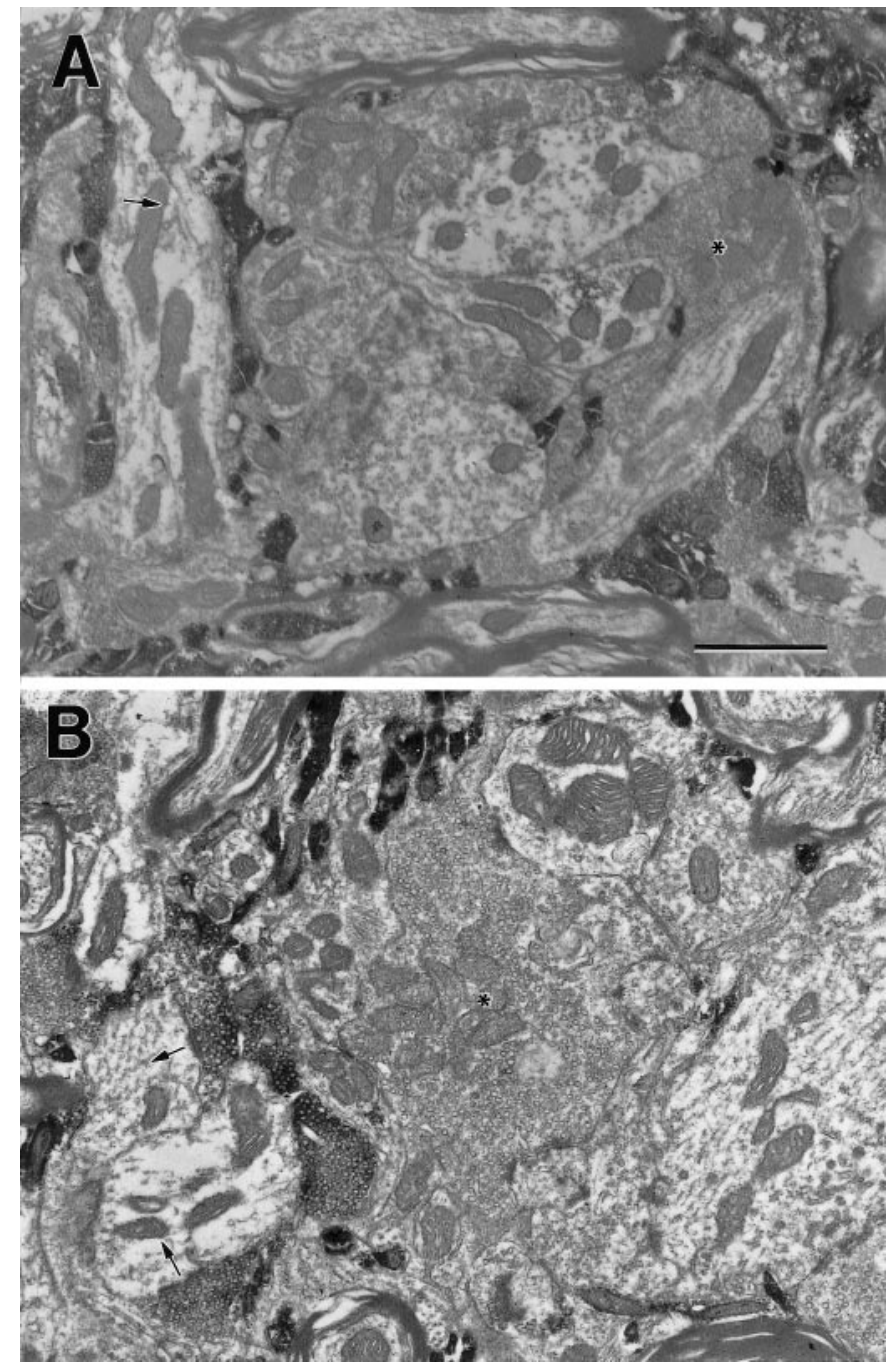

Figure 4. GAP 43 staining is excluded from glomerular arrangements. Asterisks indicate unlabeled type II terminals in glomeruli of the lateral posterior nucleus $(A)$ and the lateral geniculate nucleus $(B)$. Arrows indicate synaptic contacts made by GAP 43-stained terminals in the extraglomerular neuropil. Scale bar, $1 \mu \mathrm{m}$.

to be selectively associated with RS profiles (Godwin et al., 1997), and their activation can change the response mode of thalamocortical cells in the dLGN (McCormick and von Krosigk, 1992; Godwin et al., 1996). Thus, it appears that within the synaptic circuitry of the thalamus there is a precise localization of several proteins that can specifically amplify the signal transmitted by type I corticothalamic terminals.

Obviously, further experiments are necessary to determine the exact function of GAP 43 in the thalamus. However, the observed distribution of GAP 43 suggests that both relay and association nuclei may share a common mechanism to augment the cortical control of thalamic activity. Finally, these results underscore the distinction between the small type I corticothalamic terminals, which appear to contain GAP 43 throughout the visual thalamus, and the large type II corticothalamic terminals, which, like the type II retinal terminals in the dLGN, do not contain GAP 43.

\section{REFERENCES}

Abramson BP, Chalupa LM (1985) The laminar distribution of cortical connections with the tecto- and cortico-recipient zones in the cat's lateral posterior nucleus. Neuroscience 15:81-95. 
Baekelandt V, Arckens L, Annaer W, Eysel UT, Orban GA, Vandesande F (1994) Alterations in GAP-43 and synapsin immunoreactivity provide evidence for synaptic reorganization in adult cat dorsal lateral geniculate nucleus following retinal lesions. Eur J Neurosci 6:754-765.

Benowitz LI, Perrone-Bizzozero NI (1991) The expression of GAP-43 in relation to neuronal growth and plasticity: when, where, how and why? In: Protein kinase $\mathrm{C}$ and its brain substrates, progress in brain research, Vol 89 (Gispen WH, Routtenberg A, eds), pp 69-87. Amsterdam: Elsevier.

Benowitz LI, Routtenberg A (1997) GAP-43: an intrinsic determinant of neuronal development and plasticity. Trends Neurosci 20:84-91.

Benowitz LI, Apostolides PJ, Perrone-Bizzozero N, Finklestein SP, Zwiers H (1988) Anatomical distribution of the growth-associated protein GAP-43/B-50 in the adult rat brain. J Neurosci 8:339-352.

Bickford ME, Eisenback MA, Einstein G (1998) Synaptic targets of corticothalamic terminals in the cat pulvinar/LP complex originating from are PMLS. Soc Neurosci Abstr 24:1162.

Cucchiaro JB, Uhlrich DL, Sherman SM (1988) Parabrachial innervation of the cat's dorsal lateral geniculate nucleus: an electron microscopic study using the tracer phaseolus vulgaris leucoagglutinin (PHAL). J Neurosci 8:4576-4588.

De Graan PN, Schrama LH, Heemskerk FM, Dekker LV, Gispen WH (1990) The role of protein kinase C substrate B-50 (GAP-43) in neurotransmitter release and long-term potentiation. In: Excitatory amino acids and neuronal plasticity, advances in experimental medicine and biology, Vol 268 (Ben-Ari Y, ed), pp 347-358. New York: Plenum.

Erişir A, Van Horn SC, Bickford ME, Sherman SM (1997) Immunocytochemistry and distribution of parabrachial terminals in the lateral geniculate nucleus of the cat: a comparison with corticogeniculate terminals. J Comp Neurol 377:535-549.

Feig S, Harting JK (1998) Corticocortical communication via the thalamus: ultrastructural studies of corticothalamic projections from area 17 to the lateral posterior nucleus of the cat and inferior pulvinar nucleus of the owl monkey. J Comp Neurol 395:281-295.

Gilbert CD, Kelly JP (1975) The projections of cells in different layers of the cat's visual cortex. J Comp Neurol 163:81-106.

Godwin DW, Vaughan JW, Sherman SM (1996) Metabotropic glutamate receptors switch visual response mode of lateral geniculate nucleus cells from burst to tonic. J Neurophysiol 76:1800-1816.

Godwin DW, Van Horn SC, Erişir A, Sesma M, Romano C, Sherman SM (1997) Ultrastructural localization suggests that retinal and cortical inputs access different metabotropic glutamate receptors in the lateral geniculate nucleus. J Neurosci 16:8181-8192.

Guillery RW (1995) Anatomical evidence concerning the role of the thalamus in corticocortical communication: a brief review. Anat Embryol 187:583-592.

Jones EG, Powell TPS (1969) An electron microscopic study of the mode of termination of cortico-thalamic fibers within sensory relay nuclei of the thalamus. Proc R Soc Lond B Biol Sci 172:173-185.
Liu X-B, Jones EG (1996) Localization of alpha type II calcium calmodulin-dependent protein kinase at glutamatergic but not $\gamma$-aminobutyric acid (GABAergic) synapses in thalamus and cerebral cortex. Proc Natl Acad Sci USA 93:7332-7336.

Malenka RC, Kauer JA, Perkell DJ, Mauk MD, Kelly PT, Nicoll RA, Waxham MN (1989) An essential role for postsynaptic CaM and protein kinase activity in long-term potentiation. Nature 340:554-557.

Mathers LH (1972) The synaptic organization of the cortical projection to the pulvinar of the squirrel monkey. J Comp Neurol 146:43-60.

McCormick DA, von Krosigk M (1992) Corticothalamic activation modulates thalamic firing through glutamate "metabotropic" receptors. Proc Natl Acad Sci USA 89:2774-2778.

Neve RL, Finch EA, Bird ED, Benowitz LI (1988) Growth-associated protein GAP-43 is expressed selectively in associative regions of the adult human brain. Proc Natl Acad Sci USA 85:3638-3642.

Ogren MP, Hendrickson AE (1979) The morphology and distribution of striate cortex terminals in the inferior and lateral subdivisions of the Macaca monkey pulvinar. J Comp Neurol 188:179-200.

Ojima H, Murakami K, Kishi K (1996) Dual termination modes of corticothalamic fibers originating from pyramids of layers 5 and 6 in cat visual cortex area 17. Neurosci Lett 208:57-60.

Patel NC, Bickford ME (1997) Synaptic targets of cholinergic terminals in the pulvinar nucleus of the cat. J Comp Neurol 387:266-278.

Patel NC, Carden WB, Bickford ME (1999) Synaptic targets of cholinergic terminals in the lateral posterior nucleus of the cat. J Comp Neurol 410:31-41.

Robson JA, Hall WC (1977) The organization of the pulvinar in the grey squirrel (Sciurus carolinensis) II. Synaptic organization and comparisons with the dorsal lateral geniculate nucleus. J Comp Neurol 173:389-416.

Rockland KS (1996) Two types of corticopulvinar terminations: round (type 2) and elongate (type 1). J Comp Neurol 368:57-87.

Scharfman HE, Lu S-M, Guido W, Adams PR, Sherman SM (1990) $N$-methyl-D-aspartate (NMDA) receptors contribute to excitatory postsynaptic potentials of cat lateral geniculate neurons recorded in thalamic slices. Proc Natl Acad Sci USA 97:4548-4552.

Sherman SM, Guillery RW (1996) The functional organization of thalamocortical relays. J Neurophysiol 76:1367-1395.

Updyke BV (1975) The patterns of projection of cortical areas 17, 18, and 19 onto the laminae of the dorsal lateral geniculate nucleus in the cat. J Comp Neurol 163:377-396.

Vidnyánszky Z, Hámori J (1994) Quantitative electron microscopic analysis of synaptic input from cortical areas 17 and 18 to the dorsal lateral geniculate nucleus in cats. J Comp Neurol 349:259-268.

Vidnyánszky Z, Borostyánkoi Z, Görcs TJ, Hámori J (1996) Light and electron microscopic analysis of synaptic input from cortical area 17 to the lateral posterior nucleus in cats. Exp Brain Res 109:63-70. 